\title{
Identification of human Thioredoxin as a potent redox protein in carcinogenesis.
}

\author{
Pradhaban. $S^{1}$, Revathy. $C^{2}$ \\ ${ }^{I}$ (General surgery/ Pondicherry Institute Of Medical Sciences, Pondicherry University, India) \\ ${ }^{2}$ (Biochemistry/ Pondicherry Institute Of Medical Sciences, Pondicherry University,India )
}

\begin{abstract}
Background: Thioredoxins ("TRX") are ubiquitous 12-kDa oxidoreductase enzyme containing a dithiol-disulfide active site facilitating the reduction of other proteins by cysteine thiol-disulfide exchange. When thioredoxin levels are elevated there is increased cell growth and resistance to the normal mechanism of programmed cell death. An increase in thioredoxin levels seen in many human primary cancers compared to normal tissue appears to contribute to increased cancer cell growth and resistance to chemotherapy. Mechanisms by which thioredoxin increases cell growth include an increased supply of reducing equivalents for DNA synthesis, activation of transcription factors that regulate cell growth, and an increase in the sensitivity of cells to other cytokines and growth factors thereby thioredoxin offers a target for the development of drugs to treat and prevent cancer.
\end{abstract}

Aim: To characterize - molecular cloning, expression, purification and bioassay of the most potent redox protein in carcinogenesis.

Research methodology: The expression of thioredoxin (Trx) in human lung adenocarcinoma epithelial cells (A549) was studied by cDNA isolation using RT PCR and it was cloned into pRSET A vector at Ndel and BamH1 site (restriction sites) and then transformed in E.coli GJ1158/BL21 DE3. The cells were cultured in mass and lysed so as to generate recombinant thioredoxin, then purified to obtain a single band of appropriate molecular weight in SDS PAGE analysis. The gene sequence was confirmed by DNA sequencing and the protein by western blot using anti-thioredoxin antibody. The biological activity of recombinant thioredoxin was tested by insulin reduction assay.

Results: The biological activity of recombinant thioredoxin was studied by monitoring the dithiol-disulphide oxidoreductase activity that catalyzes reduction of insulin disulphides by dithiothreitol spectrophotometrically at $650 \mathrm{~nm}$ at $25^{\circ} \mathrm{C}$ as turbidity formation from the precipitation of the free insulin $\beta$ chain.

Keywords : cloning, protein ,E.coli, Recombinant DNA, thioredoxin,

\section{Introduction}

Thioredoxins is a $12 \mathrm{KDa}$ ubiquitous regulatory protein, which functions in maintaining cellular redox homeostasis and cell survival and is highly expressed in many cancers. The oxidative or hypoxic stress of the tumor environment resulting from abnormal angiogenesis causes unstable oxygen delivery which is known to up-regulate the expression of thioredoxin. During carcinogenesis, tumor cells often become more resistant to hypoxia or oxidative stress-induced apoptosis and most studies on tumor oxygenation have focused on these two tumor environments [1]. However, recent investigations suggest that the redox status within tumor cells determine cell survival and proliferation. Thioredoxin is known to have important roles in both these cellular responses and several studies implicate thioredoxin as a contributor to cancer progression [2].

Thioredoxins are enzymes belonging to the family of pyridine nucleotide-disulfide oxidoreductases which have a conserved CXXC a Cys-Gly-Pro-Cys redox catalytic site where the second cysteine being selenocysteine essential for activity as the truncated protein is inactive [3] and the active site of the enzyme is a selenenylsulfide/selenolthiol generated from the $\mathrm{COOH}$-terminal sequence [4]. In an oncogenic perspective, thioredoxin has been shown to regulate p53 expression and facilitate p53-dependent induction of p21 [5]. It regulates the expression of transcriptional factors such as TF111C, nuclear factor-kB, Jun/Fos, and AP-1 through redox control of these proteins, thus influencing their binding to DNA [6-8].Trx also promotes cell proliferation, and in transfection experiments, it has been shown to increase the growth rate and colony formation of nonmalignant and malignant cells [9]

\section{Research Methodology:}

Restriction enzymes and T4 DNA ligase obtained from New England Biolabs Beverley, USA. Taq DNApolymerase, Hybond nitrocellulose membranes were purchased from Amersham pharmacia biotech, Birmingham, UK. Primers used in this study were synthesized in Microsynth, Switzerland and Bangalore Genei, Bangalore, India. Large scale purification of plasmids and mini preparation kits and medias were purchased from QIAGEN, Germany. Anti Thioredoxin antibodies were purchased from Santacruz Biotechnolog, UK and 
pRSET A vector, E.coli strain BL21 DE3 and GJ1158 (expression hosts) and DH5 $\alpha$ (maintenance host), were purchased from Invitrogen Corporation, Sandiego, USA.

This study was approved by the Institutional ethical committee of the Sree Balaji Medical College and Hospital.

\section{CONSTRUCTION OF THE CLONE.}

RT-PCR was carried out and the thioredoxin gene was isolated from human adenocarcinoma epithelial cell lines (A549) and amplified using the designed forward and reverse primers (TrxF/TrxR) with Nde1 and BamH1 restriction sites.

Table 1: Primers designed for human Thioredoxin.

\begin{tabular}{|l|l|l|}
\hline Primer & Primer Sequence & Restriction enzyme \\
\hline TRXF & 5'GGGTTTCATATGGTGAAGCAGATCGAGAGCAAG3' & Nde I \\
\hline TRXR & 5'CGCGGATCCGACTAATTCATTAATGGTGGCTTC3' & Bam HI \\
\hline
\end{tabular}

The amplified gene was ligated and cloned into pRSET A vector as per manufacturer's protocol. Positive clones were selected by lysate PCR and sequenced in BIOSERV and confirmed by DNA sequencing.

Fig1: Construction of the Thioredoxin gene

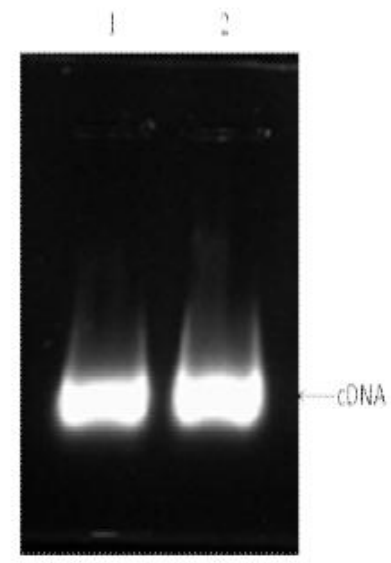

(a)

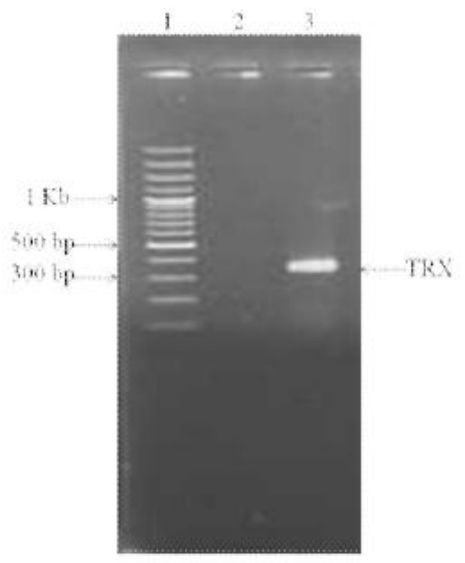

(b)

\section{Expression Of Protein In E.Coli}

The vector pRSET A based on T7 RNA polymerase was employed in the present study to express the recombinant construct in IPTG inducible BL21 (DE3) and salt inducible T7 expression host, GJ1158. The isolated thioredoxin gene was inserted into the multiple cloning sites and ampicillin resistant sequence was used for clonal selection. The protein expression was optimized and purified by chromatography using DEAE sepharose column and confirmed by western blot. The bioactivity was checked by Insulin reduction assay.

Fig 2: Expressed and purified Thioredoxin protein

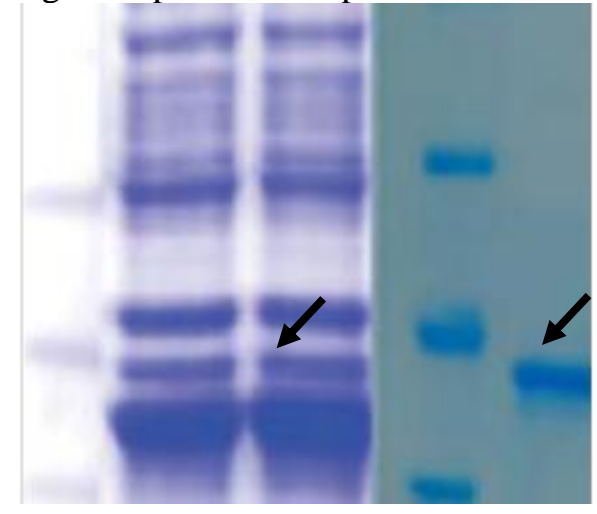

(a) SDS PAGE

\section{Thioredoxin}

Protein(12KDa)

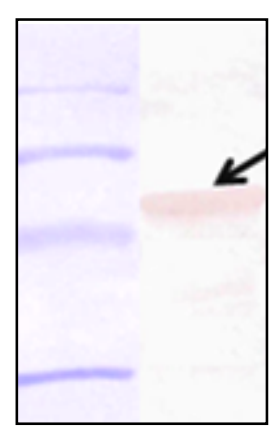

(b)Western Blot 


\section{Results:}

Thioredoxin which is encoded by 300 bp gene was amplified using gene specific primers containing Nde1 and BamH1 restriction sites in the forward and reverse primers respectively. The purified amplicons were ligated with the linearized vectors (pRSET A) using T4 DNA ligase following restriction digestion by Nde1 and BamH1 restriction enzymes. The ligation products were then transformed into DH5 $\alpha$ and the recombinants were screened by PCR and the clones were confirmed by restriction digestion. The constructs were then transformed and expressed in various E.coli strains such as BL21 (DE3) and GJ1158 and their expression profile was analyzed in SDS-PAGE.

Various parameters like growth temperature, optical density, host strain, inducer concentration etc were altered and maximum protein expression was obtained from E.coli strain GJ1158 at a temperature of $30^{\circ} \mathrm{C}$ at 0.6 optical density. Large quantities of protein generated was purified by column chromatography .The purified protein was confirmed by western blot using anti-thioredoxin antibodies.

\section{Bioassay for Thioredoxin-Insulin Reduction Assay}

Thioredoxin was shown to catalyze the reduction of insulin disulfides by dithiothreitol. An assay was developed which measures the rate of insulin reduction spectrophotometrically at $650 \mathrm{~nm}$ at $25^{\circ} \mathrm{C}$ as turbidity formation from the precipitation of the free insulin $\beta$ chain. The implication of the dithiol-disulfide oxidoreductase activity of thioredoxin for the regulation of enzyme activities by thiol oxidation-reduction control is used in this assay [10].

Insulin Reduction Assay.

Table 2: Insulin reduction assay of recombinant Thioredoxin.

\begin{tabular}{|c|c|c|c|c|}
\hline Time & B 1 & B $~$ & Positive control & Purified protien \\
\hline 0 & 0.043 & 0.040 & 0.076 & 0.045 \\
\hline 5 & 0.043 & 0.040 & 0.078 & $0-049$ \\
\hline 10 & 0.043 & $0-040$ & 0.085 & 0.051 \\
\hline 15 & 0.043 & 0.040 & 0.093 & 0.055 \\
\hline 20 & 0.043 & 0.040 & 0.101 & 0.059 \\
\hline 25 & 0.044 & 0.042 & 0.105 & 0.066 \\
\hline 30 & 0.043 & 0.041 & 0.110 & 0.075 \\
\hline 35 & 0.044 & 0.042 & 0.120 & 0.114 \\
\hline 40 & 0.043 & 0.041 & 0.127 & 0.122 \\
\hline 45 & 0.043 & 0.041 & 0.139 & 0.127 \\
\hline 50 & 0.043 & 0.041 & 0.151 & 0.141 \\
\hline 55 & 0.043 & 0.041 & 0.155 & 0.145 \\
\hline 60 & 0.043 & 0.041 & 0.158 & 0.152 \\
\hline 65 & 0.043 & 0.041 & 0.168 & 0.161 \\
\hline 70 & 0.043 & 0.041 & 0.170 & 0.170 \\
\hline 75 & 0.043 & 0.042 & 0.171 & 0.178 \\
\hline 80 & 0.044 & 0.042 & 0.173 & 0.180 \\
\hline 85 & 0.044 & 0.042 & 0.175 & 0.182 \\
\hline 90 & 0.044 & 0.042 & 0.178 & 0.184 \\
\hline 95 & 0.044 & 0.042 & 0.179 & 0.187 \\
\hline 100 & 0.044 & 0.042 & 0.184 & 0.191 \\
\hline 105 & 0.044 & 0.042 & 0.186 & 0.194 \\
\hline 110 & 0.044 & 0.042 & 0.187 & \\
\hline
\end{tabular}

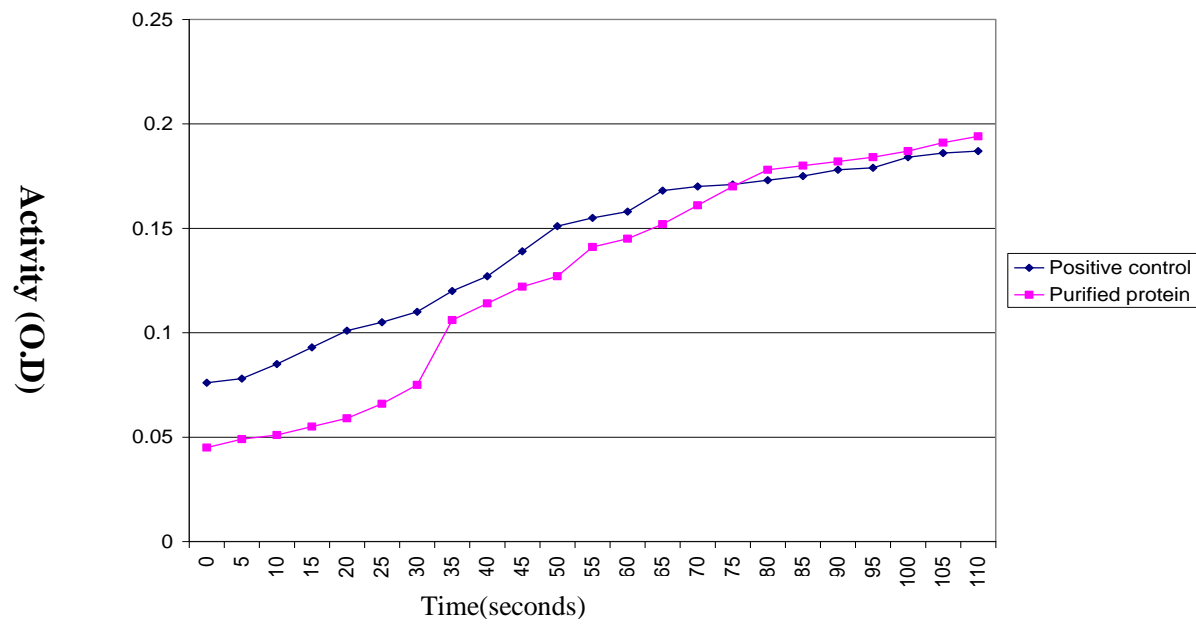

Figure3: Insulin reduction assay by Thioredoxin. 


\section{Discussion.}

Thioredoxin has an active functional role in promoting cancer cell growth and that its increased expression is not just a consequence of cancer progression. Thioredoxin is over expressed in many cancers and is considered as one of the enhancers of cancer cell growth, either through the direct stimulation of cancer cell growth or through the inhibition of cancer cell apoptosis. A recent in vivo study further highlights its importance in promoting cancer cell growth. When two human lung carcinoma cell lines, expressing either high or low Thioredoxin levels, were injected subcutaneously into SCID mice the extent of tumor growth correlated with the levels of Thioredoxin expressed by the injected cells [11]. High levels of Thioredoxin expression have also been correlated with highly invasive and metastatic tumor activity both in vitro and in vivo [12]. Thioredoxin was shown to stimulate cell invasion in these cells and to promote overall matrix metalloproteinase (MMP) activity by preferentially inhibiting the MMP inhibitors. An in vivo study using mice injected with two human carcinoma cell lines expressing either high or low levels implicates Thioredoxin as an enhancer of tumors metastasis [13]. Tumor metastases were evident in the lung of mice injected with the higher Thioredoxin expressing cell line. Similarly, expression studies have also shown the highest levels of Thioredoxin expression in the most aggressive tumors isolated from patients diagnosed with breast, melanoma, thyroid, prostate or colorectal cancer [14].

Expression of Thioredoxin also results in patients developing resistance to chemotherapeutics [15] by scavenging intracellular toxic oxidants generated by various anticancer agent [16] which suggests that Thioredoxin not only has an active role in cancer growth but also in cancer progression, through inhibition of apoptosis, stimulation of metastatic and invasive activity and through the involvement of chemotherapy resistance in cancer cells.

Considering the varied of roles for Thioredoxin in malignancy, it is ideal to explore the detailed involvement of thioredoxin in various aspects of carcinogenesis that could possibly serve as a potential targets in handling one of the most leading cause of death, the cancer[17] .

\section{References}

[1]. Therese Christina Karlenius and Kathryn Fay Tonissen, Thioredoxin and Cancer: A Role for Thioredoxin in all States of Tumor Oxygenation. Cancers 2010; 2:209-232

[2]. Ylermi Soini, Katriina Kahlos, Ulla Näpänkangas, et al. Widespread Expression of Thioredoxin and ThioredoxinReductase in Non-Small Cell Lung CarcinomaClin Cancer Res 2001; 7: 1750-1757.

[3]. Zhong, L., and Holmgren, A. Essential role of selenium in the catalytic activities of mammalian thioredoxin reductase revealed by characterization of recombinant enzymes with selenocysteine mutations.J. Biol. Chem., 275: 18121-18128, 2000.

[4]. Gasdaska, P., Oblong, J. E., Cotgreave, I. A., and Powis, G. The predicted amino acid sequence of human thioredoxin is identical to that of the autocrine growth factor human adult T-cell derived factor (ADF): thioredoxin mRNA is elevated in some human tumors. Biochim. Biophys. Acta, 1218: 292-296, 1994.

[5]. Ueno, M., Masutani, H., Arai, R. J., Yamauchi, A., Hirota, K., Sakai, T., Inamoto, T., Yamaoka, Y., Yodoi, J., and Nikaido, T. Thioredoxin-dependent redox regulation of p53-mediated p21 activation.J. Biol. Chem., 274: 35809-35815, 1999.

[6]. Matthews, J. R., Wakasugi, N., Virelizier, J. L., Yodoi, J., and Hay, R. T. Thioredoxin regulates the DNA binding activity of NF$\mathrm{kB}$ by reduction of a disulfide bond involving cysteine 62. Nucleic Acids Res. 20: 3821-3830, 1992.

[7]. Cromlish, J. A., and Roeder, R. G. Human transcription factor IIIC (TFRIC): purification, polypeptide structure, and the involvement of thiol groups in specific DNA binding. J. Biol. Chem., 264: 18100-18109, 1989

[8]. Bannister, A. J., Cook, A., and Kouzarides, T. In vitro DNA binding activity of Fos/Jun and BZFL1 but not C/EBP is affected by redox changes. Oncogene, 6: 1243-1250, 1991.

[9]. Powis, G., Kirkpatrick, D. L., Angulo, M., and Baker, A. Thioredoxin redox control of cell growth and death and the effects of inhibitors.Chem. Biol. Interact., 111-112: 23-24, 1998.

[10]. Holmgren, A. 1985. Thioredoxin. Annu. Rev. Biochem. 54:237-271.

[11]. Ceccarelli, J.; Delfino, L.; Zappia, E.; Castellani, P.; Borghi, M.; Ferrini, S.; Tosetti, F.; Rubartelli, A. The redox state of the lung cancer microenvironment depends on the levels of thioredoxin expressed by tumor cells and affects tumor progression and response to prooxidants. Int. J. Cancer 2008, 123, 1770-1778.

[12]. Farina, A.R.; Tacconelli, A.; Cappabianca, L.; Masciulli, M.P.; Holmgren, A.; Beckett, G.J.; Gulino, A.; Mackay, A.R. Thioredoxin alters the matrix metalloproteinase/tissue inhibitors of metalloproteinase balance and stimulates human SK-N-SH neuroblastoma cell invasion. Eur. J. Biochem. 2001, 268, 405-413.

[13]. Lincoln, D.T.; Ali Emadi, E.M.; Tonissen, K.F.; Clarke, F.M. The thioredoxin-thioredoxin reductase system: over-expression in human cancer. Anticancer Res. 2003, 23, 2425-2433.

[14]. Chaiswing, L.; Bourdeau-Heller, J.M.; Zhong, W.; Oberley, T.D. Characterization of redox state of two human prostate carcinoma cell lines with different degrees of aggressiveness. Free Radic. Biol. Med. 2007, 43, 202-215.

[15]. Yoshioka, J.; Schreiter, E.R.; Lee, R.T. Role of thioredoxin in cell growth through interactions with signaling molecules. Antioxid. Redox. Signal. 2006, 8, 2143-2151.

[16]. Butler, L.M.; Zhou, X.; Xu, W.S.; Scher, H.I.; Rifkind, R.A.; Marks, P.A.; Richon, V.M. The histone deacetylase inhibitor SAHA arrests cancer cell growth, up-regulates thioredoxin-binding protein-2, and down-regulates thioredoxin. Proc. Natl. Acad. Sci. USA 2002, 99, 11700-11705.

[17]. Powis, G.; Kirkpatrick, D.L. Thioredoxin signaling as a target for cancer therapy. Curr. Opin. Pharmacol. $2007,7,392-397$. 